\title{
Eigenvalue Approximation by the Finite Element Method: The Method of Lagrange Multipliers
}

\author{
By William G. Kolata
}

\begin{abstract}
The purpose of this paper is to investigate the application of the finite element method of Lagrange multipliers to the problem of approximating the eigenvalues of a selfadjoint elliptic operator satisfying Dirichlet boundary conditions. Although the Lagrange multiplier method is not a Rayleigh-Ritz-Galerkin approximation scheme, it is shown that at least asymptotically the Lagrange multiplier method has some of the properties of such a scheme. In particular, the approximate eigenvalues are greater than or equal to the exact eigenvalues and can be computed from a nonnegative definite matrix problem. It is also shown that the known estimates for the eigenvalue error are optimal.
\end{abstract}

Introduction. The application of a conventional finite element method to elliptic boundary value problems with essential boundary conditions is impractical, especially in a domain that does not have simple shape. This is due to the difficulty of constructing suitable spaces of test and trial functions that satisfy the required boundary conditions. Several techniques have been devised to bypass this difficulty, such as the least squares method of Bramble and Schatz [4], the methods of Nitsche [11], [12], and the Lagrange multiplier method of Babuška [1]. The purpose of this paper is to investigate the application of Babuška's method to the problem of approximating the eigenvalues of a selfadjoint elliptic operator satisfying Dirichlet boundary conditions.

As is common in finite element methods, the original eigenvalue problem is recast into an equivalent problem that is posed in terms of sesquilinear forms. When the original problem is selfadjoint, these forms are Hermitian. However, in the Lagrange multiplier method, these forms are not definite and so do not yield a standard Rayleigh-Ritz-Galerkin approximation scheme. Nevertheless, the Lagrange multiplier method possesses, at least asymptotically, some of the properties of a Rayleigh-RitzGalerkin scheme. In this paper it is shown that the approximate eigenvalues generated by the Lagrange multiplier method are greater than or equal to the original eigenvalues to which they are converging, provided that a convergence parameter $h$ is small enough. This is proved under certain assumptions on the rate of convergence of members of the finite element subspaces to the eigenfunctions. The proof depends

Received September 14, 1977.

AMS (MOS) subject classifications (1970). Primary 65N25, 65F 15.

Key words and phrases. Finite element method, Lagrange multipliers, selfadjoint elliptic eigenvalue problems. 
upon the construction of a lower bound for the error in the eigenvalue approximation. As a result of this lower bound, it also follows that the already known bound for the eigenvalue error, [1], [3], [13], [6] , [8], is optimal. This result may be loosely stated as follows: If the rate of convergence of the finite element subspaces to the eigenfunctions is optimal, then the rate of convergence of the approximate eigenvalues to the original eigenvalues is optimal.

At first glance, the finite dimensional eigenvalue problem associated with the Lagrange multiplier approximation presents some difficulties. The corresponding matrices are not definite. Assuming comparable accuracy, these matrices are larger $((n+m)$ $\times(n+m))$ than the matrices $(n \times n)$ that would be associated with a conventional finite element method, were such a method practical. For these reasons, a direct attack on the finite dimensional eigenvalue problem associated with the Lagrange multiplier approximation may incur unacceptable computation and storage costs. In Section 3 of this paper an alternate approach to the solution of the finite dimensional problem is outlined. In this approach the approximate eigenvalues and eigenvectors of the Lagrange multiplier method are computed using an associated $n \times n$ nonnegative definite matrix. A scheme for constructing this matrix is presented.

1. Eigenvalue Approximation by the Lagrange Multiplier Method. The focus of this paper is on the approximation of the eigenvalues of the following selfadjoint elliptic eigenvalue problem:

$$
\begin{array}{rr}
-\Delta u+c u=\omega u & \text { in } \Omega, \\
u=0 & \text { on } \Gamma,
\end{array}
$$

where $\Omega$ is a bounded region in the plane with smooth boundary $\Gamma, \omega$ is the eigenvalue parameter, $c$ is a smooth, real valued function on $\bar{\Omega}$, and, without loss of generality, $c_{0}=\sup _{x \in \bar{\Omega}}|c(x)|>0$. The results that follow can easily be extended to more general 2 nd order elliptic operators in more general bounded domains.

The method of Lagrange multipliers is based on a formulation of (1.1), (1.2) in terms of sesquilinear forms [1] , [2] , [7]. Let $H$ be the complex Hilbert space $H^{1}(\Omega)$ $\oplus H^{-1 / 2}(\Gamma)$ with norm $\|\cdot\|^{2}=\|\cdot\|_{1}^{2}+|\cdot|_{-1 / 2}^{2}$, where $H^{1}(\Omega)$ and $H^{-1 / 2}(\Gamma)$ are the standard Sobolev spaces [10]. For any $u, v$ in $H^{1}(\Omega)$, set

$$
a(u, v)=\int_{\Omega}(\nabla u \cdot \nabla \bar{v}+c u \bar{v}) d x .
$$

For any $U=(u, \lambda), V=(v, \mu)$ in $H$, set $A(U, V)=a(u, v)-\int_{\Gamma}(\lambda \bar{v}+\bar{\mu} u) d s$, and $B(U, V)=\int_{\Omega} u \bar{v} d x$. Then $A(\cdot, \cdot)$ and $B(\cdot, \cdot)$ are bounded, Hermitian, sesquilinear forms on $H \times H$. Neither $A(\cdot, \cdot)$ nor $B(\cdot, \cdot)$ are definite, but $A(\cdot, \cdot)$ satisfies

$$
\inf _{\substack{U \in H \\\|U\|=1}} \sup _{\substack{V \in H \\ V \|=1}}|A(U, V)| \geqslant \alpha>0, \quad \text { [2]. }
$$

Problem (1.1), (1.2) can be formulated as:

$$
A(U, V)=\omega B(U, V) \text { for all } v \in H, U \neq 0 .
$$

By (1.3), there is a bounded linear operator $T$ on $H$ such that $A(T U, V)=B(U, V)$ 
for all $V$ in $H$. The operator $T$ is also a bounded map from $H$ into $H^{3}(\Omega) \oplus H^{3 / 2}(\Gamma)$ and so by Rellich's theorem is compact on $H$. In fact, if $T U=(\varphi, \lambda)$, then $\varphi$ is a weak solution of $-\Delta \varphi+c \varphi=u ; \varphi=0$ on $\Gamma$. Moreover, $\lambda=\partial \varphi / \partial n$, [1]. By regularity theorems, $\varphi$ is in $H^{3}(\Omega)$ and $\lambda=\partial \varphi / \partial n$ is in $H^{3 / 2}(\Gamma),[10]$. It is easily seen that the eigenvalues of (1.4) are the reciprocals of the eigenvalues of the operator $T$ and the associated eigenvectors are identical. Since $A(U, U)>0$ for any eigenvector $U, T$ has no generalized eigenvectors. Furthermore, again by regularity, the eigenvalues and eigenvectors of (1.1), (1.2) are the same as those of (1.4).

Let $S_{h_{\Omega}}(\Omega) \subseteq H^{1}(\Omega)$ and $S_{h_{\Gamma}}(\Gamma) \subseteq H^{1 / 2}(\Gamma)$ be finite dimensional subspaces. Set $S_{h}=S_{h_{\Omega}}(\Omega) \oplus S_{h_{\Gamma}}(\Gamma)$, where $h_{\Omega}^{-2}, h_{\Gamma}^{-1}$, and $h^{-2}=h_{\Omega}^{-2}+h_{\Gamma}^{-1}$ denote the dimensions of the corresponding subspaces. To simplify notation set $S_{h}(\Omega) \equiv S_{h_{\Omega}}(\Omega)$ and $S_{h}(\Gamma) \equiv S_{h_{\Gamma}}(\Gamma)$. With the aid of these subspaces, a Galerkin approximation for the eigenvalue problem (1.4) can be constructed:

$$
A\left(U_{h}, V_{h}\right)=\omega_{h} B\left(U_{h}, V_{h}\right) \text { for all } V_{h} \in S_{h}, U_{h} \neq 0 .
$$

This reduces to a matrix eigenvalue problem when a basis $\left(\varphi_{1}(x), 0\right), \ldots,\left(\varphi_{n}(x), 0\right)$, $\left(0, \psi_{1}(s)\right), \ldots,\left(0, \psi_{m}(s)\right)$ is chosen for $S_{h}$, and $U_{h}$ is set equal to $\left(u_{h}, \lambda_{h}\right)=$ $\left(\sum_{j=1}^{n} u_{j} \varphi_{j}(x), \Sigma_{i=1}^{m} \lambda_{i} \psi_{i}(s)\right)$, where $n=h_{\Omega}^{-2}$ and $m=h_{\Gamma}^{-1}$. Problem (1.5) is then equivalent to:

$$
\left[\begin{array}{ll}
K & M \\
M^{H} & 0
\end{array}\right]\left[\begin{array}{l}
u \\
\lambda
\end{array}\right]=\omega_{h}\left[\begin{array}{ll}
B & 0 \\
0 & 0
\end{array}\right]\left[\begin{array}{l}
u \\
\lambda
\end{array}\right],
$$

where $K$ is the $n \times n$ matrix with $k_{i j}=a\left(\varphi_{i}, \varphi_{j}\right), M$ is the $n \times m$ matrix with $m_{i, k}=$ $-\int_{\Gamma} \bar{\varphi}_{i} \psi_{k} d s, B$ is the $n \times n$ matrix with $b_{i j}=\int_{\Omega} \varphi_{i} \bar{\varphi}_{j} d x$, and $u=\left(u_{j}\right)^{T}, \lambda=\left(\lambda_{k}\right)^{T}$ are, respectively, $n$ - and $m$-dimensional column vectors. The solutions of (1.6) will be discussed in Section 3.

How closely the eigenvalues and eigenvectors of (1.6) approximate those of (1.4) depends on how closely the members of the subspace $S_{h}$ approximates the eigenvectors of (1.4). Suppose then that there is a number $k>1$ and a positive constant $C_{\Omega}$, independent of $h_{\Omega}$, such that for any $v \in H^{s}(\Omega)$ there is a $v_{h} \in S_{h}(\Omega)$ with

$$
\left\|v-v_{h}\right\|_{q} \leqslant C_{\Omega} h_{\Omega}^{s-q}\|v\|_{s} \text { for } 0 \leqslant q \leqslant s \leqslant k .
$$

Similarly, suppose there is a number $m>1 / 2$ and a constant $C_{\Gamma}$, independent of $h_{\Gamma}$, such that for any $\mu \in H^{t}(\Gamma)$ there is a $\mu_{h} \in S_{h}(\Gamma)$ with

$$
\left|\mu-\mu_{h}\right|_{p} \leqslant C_{\Gamma} h_{\Gamma}^{t-p}|\mu|_{t} \text { for }-1 / 2 \leqslant p \leqslant 1 / 2 \leqslant t \leqslant m .
$$

Assume also that $S_{h}(\Gamma)$ satisfies the "inverse assumption"

$$
\left|\mu_{h}\right|_{1 / 2} \leqslant C h_{\Gamma}^{-1}\left|\mu_{h}\right|_{-1 / 2}
$$

where $C$ is a positive constant independent of $h_{\Gamma}$. For a discussion of properties (1.7)-(1.9) and some examples, see [2], [3] .

LEMMA 1. If $h_{\Omega} / h_{\Gamma}$ is small enough, then for $h$ sufficiently small there is a 


\section{positive constant $\alpha_{0}$ independent of $h$ such that}

$$
\inf _{\substack{U_{h} \in S_{h} \\\left\|U_{h}\right\|=1}} \sup _{\substack{V_{h} \in S_{h} \\\left\|V_{h}\right\|=1}}\left|A\left(U_{h}, V_{h}\right)\right| \geqslant \alpha_{0} .
$$

Proof. See [2], [7].

Lemma 1 ensures the existence of the projection $P_{h}$ of $H$ onto $S_{h}$ defined by the relation $A\left(P_{h} U, V_{h}\right)=A\left(U, V_{h}\right)$ for all $V_{h} \in S_{h}$. Suppose $C_{1}$ is the constant given by $|A(U, V)| \leqslant C_{1}\|U\|\|V\|$. Then, $\left\|P_{h}\right\| \leqslant C_{1} / \alpha_{0}$ and

$$
\left\|U-P_{h} U\right\| \leqslant\left(1+\frac{C_{1}}{\alpha_{0}}\right) \inf _{U_{h} \in S_{h}}\left\|U-U_{h}\right\|, \quad \text { [2]. }
$$

Set $T_{h}=P_{h} T$. Then, $T_{h}$ is a bounded operator on $H$ with range in $S_{h}$ and satisfies $A\left(T_{h} U, V_{h}\right)=A\left(T U, V_{h}\right)=B\left(U, V_{h}\right)$ for all $V_{h} \in S_{h}$. Thus,

$$
A\left(\left(T-T_{h}\right) U, V_{h}\right)=0 \text { for all } V_{h} \in S_{h} .
$$

The eigenvalues of $T_{h}$ are the reciprocals of the eigenvalues of (1.5) (or (1.6)) and the eigenvectors are identical. It will be shown in Section 3 that the eigenvalues of (1.6) are positive real numbers and each has ascent 1 , i.e., there are no generalized eigenvectors. The same then must hold for the eigenvalues of $T_{h}$. Let $C_{3}$ denote the norm of $T$ as a map from $H$ into $H^{3}(\Omega) \oplus H^{3 / 2}(\Gamma)$. Then, by (1.7), (1.8), and (1.11),

$$
\left\|T-T_{h}\right\| \leqslant\left(1+\frac{C_{1}}{\alpha_{0}}\right) C_{3}\left(C_{\Omega}^{2} h_{\Omega}^{2 p-2}+C_{\Gamma}^{2} h_{\Gamma}^{2 q+1}\right)^{1 / 2},
$$

where $p=\min (k, 3)$ and $q=\min (m, 3 / 2)$. Thus, $T_{h} \rightarrow T$ in norm.

Since $T$ is compact and $T_{h} \rightarrow T$ in norm, the eigenvalues and corresponding eigenvectors of $T_{h}$ converge to eigenvalues and corresponding eigenvectors of $T$. In fact, suppose $\xi$ is an eigenvalue of $T$ with multiplicity $m$. Let $C$ be a circle centered at $\xi$ contained in the resolvent set, $\rho(T)$, of $T$ and enclosing no other eigenvalue of $T$. Then, $E \equiv E(\xi) \equiv(1 / 2 \pi i) \int_{C}(z-T)^{-1} d z$ is a bounded projection with range $R(E)=$ space of eigenvectors of $T$ associated with $\xi$. For $h$ sufficiently small, $C \subseteq \rho\left(T_{h}\right), E_{h}$ $\equiv E_{h}(\xi)=(1 / 2 \pi i) \int_{C}\left(z-T_{h}\right)^{-1} d z$ is a projection onto its range $R\left(E_{h}\right)$, and $\left\|E-E_{h}\right\|$ $\longrightarrow 0$ as $h \longrightarrow 0$. The dimension of $R\left(E_{h}\right)$ is $m$, the same as that of $R(E)$, and $R\left(E_{h}\right)$ is the direct sum of the spaces of eigenvectors of $T_{h}$ corresponding to eigenvalues enclosed by $C$. Thus, counting according to multiplicity, there are $m$ such eigenvalues, denoted $\xi_{1}(h), \ldots, \xi_{m}(h)$. These eigenvalues converge to $\xi$ as $h \rightarrow 0$, and the subspaces $R(E), R\left(E_{h}\right)$ approach one another. These facts may be found in [5].

To end this section, a few results that will be needed later are listed. For $h$ sufficiently small, the restriction of $E_{h}$ to $R(E), E_{\left.h\right|_{R(E)}}$, is a surjection of $R(E)$ onto $R\left(E_{h}\right)$. Thus, $E_{\left.h\right|_{R(E)}}$ has an inverse, denoted $E_{h}^{-1}$, which maps $R\left(E_{h}\right)$ onto $R(E)$. It follows that $L_{h} \equiv E_{h}^{-1} E_{h}$ is the identity on $R(E)$ and is a projection of $H$ onto $R(E)$ along the null space of $E_{h}, N\left(E_{h}\right)$. The norms $\left\|E_{h}\right\|$ and $\left\|E_{h}^{-1}\right\|$ are bounded independently of $h$. Let $\hat{T}_{h}$ be the operator on $R(E)$ defined by $\hat{T}_{h} \equiv E_{h}^{-1} T_{h} E_{\left.h\right|_{R}(E)}$. Because $R\left(E_{h}\right)$ is an invariant subspace for $T_{h}$, the eigenvalues of $\hat{T}_{h}$ are $\xi_{1}(h), \ldots$, 
$\xi_{m}(h)$, and the multiplicity of $\xi_{i}(h)$ as an eigenvalue of $\hat{T}_{h}$ equals the multiplicity of $\xi_{i}(h)$ as an eigenvalue of $T_{h}$. Let $\hat{T}$ be the restriction of $T$ to $R(E)$. Then $\hat{T}$ has $\xi$ as its only eigenvalue and $\xi$ has multiplicity $m$. Because $L_{h}$ is the identity on $R(E)$, for any $\Phi \in R(E)$,

$$
\left(\hat{T}-\hat{T}_{h}\right) \Phi=L_{h}\left(T-T_{h}\right) \Phi .
$$

These results can be found in [13].

Given any bounded operator $R$ on $H$, by (1.3), there exists a unique bounded operator $R_{*}$ such that $A(R U, V)=A\left(U, R_{*} V\right)$. Because in this case $A(\cdot, \cdot)$ are hermitian, it follows that $T_{*}=T$ and $P_{h *}=P_{h}$ and so $\left(T_{h}\right)_{*}=\left(P_{h} T\right)_{*}=T P_{h}$. In addition, for any $U \in H$

$$
\left\|\left(T_{*}-T_{h *}\right) U\right\|=\left\|\left(T-T P_{h}\right) U\right\| \leqslant \frac{C_{1}}{\alpha}\left\|T-T_{h}\right\| \inf _{U_{h} \in S_{h}}\left\|U-U_{h}\right\| .
$$

These results can be found in [8].

2. Estimates for the Error in the Eigenvalue Approximation. Let $\xi$ be an eigenvalue of $T$ with $R(E)$ the associated space of eigenvectors. Assume $\xi$ has multiplicity $m$ and so $\operatorname{dim} R(E)=m$. Then $\omega=1 / \xi$ is an eigenvalue of (1.1), (1.2) and $\varphi$ is an associated eigenvector if and only if $\Phi=(\varphi, \partial \varphi / \partial n)$ is in $R(E)$. For the remainder of this section $\xi$ and $R(E)$ will be fixed.

Suppose $\xi_{1}(h), \ldots, \xi_{m}(h)$ are the eigenvalues of $T_{h}$ converging to $\xi$. Then $\omega_{1}(h), \ldots, \omega_{m}(h)$, where $\omega_{i}(h)=\xi_{i}(h)^{-1}$, are the eigenvalues of (1.5) (or (1.6)) converging to $\omega$. The eigenvectors of $T_{h}$ associated with $\xi_{1}(h), \ldots, \xi_{m}(h)$ are also the eigenvectors of (1.5) or (1.6) associated with $\omega_{1}(h), \ldots, \omega_{m}(h)$. The estimates for the eigenvalue and eigenvector error can be expressed in terms of the quantity

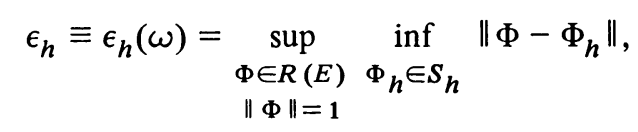

which measures how well the elements of $R(E)$ can be approximated by elements from $S_{h}$. The following two theorems give estimates for the eigenvalue and eigenvector approximation.

THEOREM 1. For h sufficiently small, there is a positive constant $K_{1}$, depending on $R(E)$ but not on $h$, such that for each $i=1, \ldots, m$

$$
\left|\xi-\xi_{i}(h)\right|=\left|\frac{\omega_{i}(h)-\omega}{\omega_{i}(h) \omega}\right| \leqslant K_{1} \epsilon_{h}^{2}
$$

Proof. See [1], [3], [13], [6], and [8].

THEOREM 2. For each $h$, let $U_{i}(h)$ be an eigenvector corresponding to $\xi_{i}(h)=$ $\omega_{i}(h)^{-1}$ such that $\left\|U_{i}(h)\right\|=1$. Then there is an eigenvector $\Phi_{i}(h)$ in $R(E)$ and a constant $K_{2}$, depending on $R(E)$ but not on $h$, such that

$$
\left\|\Phi_{i}(h)-U_{i}(h)\right\| \leqslant K_{2} \epsilon_{h} .
$$

Proof. See [1], [3], [13], [6], and [8]. 
The remainder of this section is devoted to constructing a lower bound for $\xi$ $\xi_{i}(h)$ and discussing some of the consequences of this lower bound.

LEMma 3. For each $\xi_{i}(h)$ there is a $\Phi_{i}(h)$ in $R(E)$ such that $A\left(\Phi_{i}(h), \Phi_{i}(h)\right)=1$ and

$$
\xi-\xi_{i}(h)=A\left(\left(\hat{T}-\hat{T}_{h}\right) \Phi_{i}(h), \Phi_{i}(h)\right)
$$

Proof. Recall that $\xi_{i}(h)$ is also an eigenvalue of $\hat{T}_{h}$. Let

$$
\Phi_{i}(h) \equiv\left(\varphi_{i}(h), \frac{\partial \varphi_{i}}{\partial n}(h)\right) \equiv\left(\varphi_{i}(h), \lambda_{i}(h)\right) \in R(E)
$$

be a corresponding eigenvector normalized so that $a\left(\varphi_{i}(h), \varphi_{i}(h)\right)=1$. Then,

$$
A\left(\Phi_{i}(h), \Phi_{i}(h)\right)=a\left(\varphi_{i}(h), \varphi_{i}(h)\right)-\int_{\Gamma}\left(\lambda_{i}(h) \bar{\varphi}_{i}(h)+\bar{\lambda}_{i}(h) \varphi_{i}(h)\right) d s=1,
$$

since $\varphi_{i}(h)$ is an eigenvector of $(1.1),(1.2)$ associated with $\omega=\xi^{-1}$ and so $\varphi_{i}(h)=$ 0 on $\Gamma$.

Now

$$
\begin{aligned}
\xi-\xi_{i}(h) & =A\left(\left(\xi-\xi_{i}(h)\right) \Phi_{i}(h), \Phi_{i}(h)\right) \\
& =A\left(\left\{\xi-\xi_{i}(h)-(\xi-T)\right\} \Phi_{i}(h), \Phi_{i}(h)\right) \\
& =A\left(\left(T-\xi_{i}(h)\right) \Phi_{i}(h), \Phi_{i}(h)\right)=A\left(\left(\hat{T}-\hat{T}_{h}\right) \Phi_{i}(h), \Phi_{i}(h)\right),
\end{aligned}
$$

since $\hat{T}=T_{\mid R(E)}$ and $\hat{T}_{h} \Phi_{i}(h)=\xi_{i}(h) \Phi_{i}(h)$.

Lemma 4. Suppose $\Phi=(\varphi, \partial \varphi / \partial n) \in R(E)$ and $A(\Phi, \Phi)=a(\varphi, \varphi)=1$. Then,

$$
\|\Phi\| \leqslant \kappa_{0} \omega \equiv\left\{\frac{\left(1+\hat{C}_{\tau}^{2}\right)^{1 / 2} C_{3}^{\prime}}{\beta_{0}^{1 / 2}}\right\} \omega,
$$

where $\beta_{0}=\min \left(1, c_{0}\right), \hat{C}_{\tau}$ is the trace constant for the map $\partial / \partial n: H^{3}(\Omega) \rightarrow$ $H^{3 / 2}(\Gamma),[9]$, and $C_{3}^{\prime}$ is a positive constant that does not depend on $\omega$.

Proof. Let $S$ be the map from $H^{0}(\Omega)$ into $H_{0}^{1}(\Omega)$ defined by $a(S f, v)=(f, v)$ for all $v$ in $H_{0}^{1}(\Omega)$. The map $S$ is well defined since $a(u, u) \geqslant \beta_{0}\|u\|_{1}^{2}$; moreover by results on regularity [10], $S$ is a bounded map from $H^{1}(\Omega)$ into $H^{3}(\Omega) \cap H_{0}^{1}(\Omega)$. Let $C_{3}^{\prime}$ be the norm of $S$ as map from $H^{1}(\Omega)$ into $H^{3}(\Omega)$. Again by regularity, $\omega$ is an eigenvalue of (1.1), (1.2) if and only if $\xi=1 / \omega$ is an eigenvalue of $S$ considered as an operator on $H^{0}(\Omega)$, and $\varphi$ is an associated eigenvector if and only if it is an eigenvector of $S$. Thus,

$$
\begin{aligned}
\|\Phi\|^{2} & =\|\varphi\|_{1}^{2}+\left|\frac{\partial \varphi}{\partial n}\right|_{-1 / 2}^{2} \leqslant\|\varphi\|_{3}^{2}+\left|\frac{\partial \varphi}{\partial n}\right|_{3 / 2}^{2} \leqslant\left(1+\hat{C}_{\tau}^{2}\right)\|\varphi\|_{3}^{2} \\
& =\left(1+\hat{C}_{\tau}^{2}\right) \frac{\|S \varphi\|_{3}^{2}}{\xi^{2}}=\left(1+\hat{C}_{\tau}^{2}\right) \omega^{2}\|S \varphi\|_{3}^{2} \leqslant\left(1+\hat{C}_{\tau}^{2}\right) \omega^{2}\left(C_{3}^{\prime}\right)^{2}\|\varphi\|_{1}^{2} \\
& \leqslant \frac{\left(1+\hat{C}_{\tau}^{2}\right)\left(\omega^{2}\left(C_{3}^{\prime}\right)^{2}\right.}{\beta_{0}} a(\varphi, \varphi)=\frac{\left(1+\hat{C}_{\tau}^{2}\right)\left(C_{3}^{\prime}\right)^{2} \omega^{2}}{\beta_{0}} .
\end{aligned}
$$


Estimate (2.5) follows.

Lemma 5. Let $\Phi=(\varphi, \partial \varphi / \partial n) \in R(E)$ with $A(\Phi, \Phi)=a(\varphi, \varphi)=1$. Then, for $h$ sufficiently small, there is a constant $C_{L}$, depending on $R(E)$ but not on $h$, such that

$$
\left\|L_{h *} \Phi-\Phi\right\| \leqslant C_{L}\left\|T-T_{h}\right\| \epsilon_{h} .
$$

Moreover,

$$
\left\|\left(T-T_{h}\right) \Phi\right\| \leqslant \kappa_{0}\left(1+C_{1} / \alpha_{0}\right) \epsilon_{h} .
$$

Proof. Estimate (2.6) follows from Lemmas 4 and 5 of [8], which in turn follow from lemmas of [13]. The constant $C_{L}$ is equal to $\kappa_{0} \omega C_{1} C_{L}^{\prime} / \alpha_{0}$ and $C_{L}^{\prime}$ is a constant depending on $\|E\|$, $\sup _{z \in C}\left\|(z-T)^{-1}\right\|, \sup _{h ; z \in C}\left\|\left(z-T_{h *}\right)^{-1}\right\|$ and the length of $C$ where $C$ is the contour about $\xi$ defining $E$.

To prove (2.7) set $\widetilde{\Phi}=\Phi /\|\Phi\|$ and note that

$$
\begin{aligned}
\left\|\left(T-T_{h}\right) \Phi\right\| & =\left\|\left(I-P_{h}\right) T \Phi\right\|=\xi\left\|\left(I-P_{h}\right) \Phi\right\|=\xi\|\Phi\|\left\|\left(I-P_{h}\right) \tilde{\Phi}\right\| \\
& \leqslant \xi \kappa_{0} \omega\left(1+\frac{C_{1}}{\alpha_{0}}\right) \inf _{\Phi_{h} \in S_{h}}\left\|\widetilde{\Phi}-\Phi_{h}\right\|
\end{aligned}
$$

by (1.11) and (2.5). Estimate (2.7) follows immediately from the definition of $\epsilon_{h}$.

LEMMA 6. Let $\xi_{i}(h)$ be one of the eigenvalues converging to $\xi$ and let $\Phi_{i}(h) \equiv$ $\Phi \equiv(\varphi, \partial \varphi / \partial n) \equiv(\varphi, \lambda)$ be the eigenvector associated with $\xi_{i}(h)$ as in Lemma 3. (The indices $i$ and $h$ on $\Phi$ are suppressed and should be understood.) Suppose there is a positive function $w(h)$ such that $w(h) \rightarrow 0$ as $h \rightarrow 0$ and

$$
\inf _{\eta \in S_{h}(\Gamma)}|\lambda-\eta|_{-1 / 2} \leqslant w(h)\left\|\varphi-\varphi_{h}\right\|_{1},
$$

for $h$ sufficiently small, where $\left(\varphi_{h}, \lambda_{h}\right)=P_{h} \Phi=\Phi_{h}$. Then there are positive functions $\beta_{0}(h)$ and $\beta_{1}(h)$, with $\beta_{0}(h) \rightarrow \beta_{0}$ and $\beta_{1}(h) \longrightarrow 0$ as $h \longrightarrow 0$, such that, for $h$ sufficiently small,

$$
\xi-\xi_{i}(h) \geqslant \xi\left\{\beta_{0}(h)\left\|\varphi-\varphi_{h}\right\|_{1}^{2}-\beta_{1}(h) \epsilon_{h}^{2}\right\} .
$$

Proof. By Lemma 3 and (1.14),

$$
\begin{aligned}
\xi-\xi_{i}(h) & =A\left(\left(\hat{T}-\hat{T}_{h}\right) \Phi, \Phi\right)=A\left(L_{h}\left(T-T_{h}\right) \Phi, \Phi\right) \\
& =A\left(\left(T-T_{h}\right) \Phi, \Phi\right)+A\left(\left(T-T_{h}\right) \Phi, L_{h *} \Phi-\Phi\right) .
\end{aligned}
$$

Now, by (1.12),

$$
\begin{aligned}
A\left(\left(T-T_{h}\right) \Phi, \Phi\right) & =\xi^{-1} A\left(\left(T-T_{h}\right) \Phi, T \Phi\right)=\xi^{-1} A\left(\left(T-T_{h}\right) \Phi,\left(T-T_{h}\right) \Phi\right) \\
& =\xi A\left(\left(I-P_{h}\right) \Phi,\left(I-P_{h}\right) \Phi\right) \\
& =\xi\left\{a\left(\varphi-\varphi_{n}, \varphi-\varphi_{n}\right)-2 \operatorname{Re} \int_{\Gamma}\left(\lambda-\lambda_{h}\right)\left(\overline{\varphi-\varphi_{h}}\right) d s\right\} .
\end{aligned}
$$

By the definition of $P_{h}, A\left(\Phi-\Phi_{h}, V_{h}\right)=0$ for any $V_{h}=\left(v_{h}, \mu_{h}\right)$ in $S_{h}$. 
Choose $V_{h}=(0, \eta)$ for any $\eta \in S_{h}(\Gamma)$. It follows that $\int\left(\varphi-\varphi_{h}\right) \bar{\eta} d s=0$. In particular, since $\lambda_{h} \in S_{h}(\Gamma)$,

$$
\int_{\Gamma}\left(\lambda-\lambda_{h}\right)\left(\overline{\varphi-\varphi_{h}}\right) d s=\int_{\Gamma}(\lambda-\eta)\left(\overline{\varphi-\varphi_{h}}\right) d s=\int_{\Gamma} \lambda\left(\overline{\left.\varphi-\varphi_{h}\right)} d s\right.
$$

any $\eta \in S_{h}(\Gamma)$. Thus, replacing $\lambda_{h}$ in the last expression of the previous paragraph by an arbitrary $\eta \in S_{h}(\Gamma)$ yields

$$
\xi\left\{a\left(\varphi-\varphi_{h}, \varphi-\varphi_{h}\right)-2 \operatorname{Re} \int_{\Gamma}(\lambda-\eta)\left(\overline{\varphi-\varphi_{h}}\right) d s\right\} \quad \text { for any } \eta \in S_{h}(\Gamma) \text {. }
$$

This expression in turn can be estimated below as follows.

$$
\begin{aligned}
\xi\left\{a\left(\varphi-\varphi_{h}, \varphi-\varphi_{h}\right)-2 \operatorname{Re} \int_{\Gamma}\right. & \left.(\lambda-\eta)\left(\overline{\varphi-\varphi_{h}}\right) d s\right\} \\
& \geqslant \xi\left\{\beta_{0}\left\|\varphi-\varphi_{h}\right\|_{1}^{2}-2|\lambda-\eta|_{-1 / 2}\left|\varphi-\varphi_{h}\right|_{1 / 2}\right\} \\
& \geqslant \xi\left\{\beta_{0}\left\|\varphi-\varphi_{h}\right\|_{1}^{2}-2 C_{\tau}|\lambda-\eta|_{-1 / 2}\left\|\varphi-\varphi_{h}\right\|_{1}\right\},
\end{aligned}
$$

where $C_{\tau}$ is the bound for the trace map $u \in H^{1}(\Omega) \stackrel{\tau}{\longrightarrow} u_{\mid \Gamma} \in H^{1 / 2}(\Gamma)$. Because $\eta$ is arbitrary, it can be chosen so that by (2.8) this last term can be bounded below by $\xi\left\{\beta_{0}-2 C_{\tau} w(h)\right\}\left\|\varphi-\varphi_{h}\right\|_{1}^{2}$. Consequently, $A\left(\left(T-T_{h}\right) \Phi, \Phi\right) \geqslant \xi \beta_{0}(h)\left\|\varphi-\varphi_{h}\right\|_{1}^{2}$, where $\beta_{0}(h)=\beta_{0}-2 C_{\tau} w(h) \rightarrow \beta_{0}$ as $h \rightarrow 0$.

On the other hand,

$$
\begin{aligned}
A\left(\left(T-T_{h}\right) \Phi, L_{h *} \Phi-\Phi\right) & \leqslant C_{1}\left\|\left(T-T_{h}\right) \Phi\right\|\left\|L_{h *} \Phi-\Phi\right\| \\
& \leqslant C_{1} C_{L} \kappa_{0}\left(1+C_{1} / \alpha_{0}\right)\left\|T-T_{h}\right\| \epsilon_{h}^{2}
\end{aligned}
$$

by Lemma 5. Set $\beta_{1}(h)=\xi^{-1} C_{1} C_{L^{\kappa}}{ }_{0}\left(1+C_{1} / \alpha_{0}\right)\left\|T-T_{h}\right\|$. With $\beta_{0}(h)$ and $\beta_{1}(h)$ so defined, (2.9) follows.

THEOREM 3. Suppose that for any $\Phi=(\varphi, \lambda) \in R(E)$,

$$
\begin{aligned}
& \inf _{x \in S_{h}(\Omega)}\|\varphi-\chi\|_{1} \geqslant \hat{C}_{\Omega} h_{\Omega}^{k-1}\|\varphi\|_{k} ; \\
& \inf _{\eta \in S_{h}(\Gamma)}|\lambda-\eta|_{-1 / 2} \geqslant \hat{C}_{\Gamma} h_{\Gamma}^{m+1 / 2}|\lambda|_{m},
\end{aligned}
$$

where $\hat{C}_{\Omega}, \hat{C}_{\Gamma}$ are positive constants depending perhaps on $R(E)$ but independent of $h$. Suppose also that there is an $s>0$ and a positive constant $C_{s}$ independent of $h$ such that

$$
h_{\Omega} / h_{\Gamma} \geqslant C_{s} h_{\Gamma}^{s}
$$

Then if $k+1 / 2 \geqslant m>k-3 / 2+s(k-1)$, there is a positive constant $\beta_{3}$, depending on $R(E)$ but not $h$, such that for $h$ sufficiently small

$$
\frac{\xi-\xi_{i}(h)}{\xi}=\frac{\omega_{i}(h)-\omega}{\omega_{i}(h)} \geqslant \beta_{3} \epsilon_{h}^{2}
$$


Proof. The first step in the proof is to show that (2.10) and (2.11) imply (2.8). Suppose then that $\Phi \equiv \Phi_{i}(h) \equiv(\varphi, \lambda)$ is chosen as in Lemma 3 and set $P_{h} \Phi=$ $P_{h} \Phi_{i}(h)=\left(\varphi_{h}, \lambda_{h}\right)$. Then, by $(1.8)$ and $(2.10)$,

$$
\begin{aligned}
\inf _{\eta \in S_{h}(\Gamma)}|\lambda-\eta|_{-1 / 2} & \leqslant C_{\Gamma} h_{\Gamma}^{m+1 / 2}|\lambda|_{m}=\left\{\frac{C_{\Gamma} h_{\Gamma}^{m+1 / 2}|\lambda|_{m}}{\hat{C}_{\Omega} h_{\Omega}^{k-1}\|\varphi\|_{k}}\right\} \hat{C}_{\Omega} h_{\Omega}^{k-1}\left\|_{\varphi}\right\|_{k} \\
& \leqslant w(h) \inf _{\chi \in S_{h}(\Omega)}\|\varphi-\chi\|_{1},
\end{aligned}
$$

where $w(h)=C_{\Gamma} h_{\Gamma}^{m+1 / 2}|\lambda|_{m} / \hat{C}_{\Omega} h_{\Omega}^{k-1}\|\varphi\|_{k}$. By (2.11)

$$
\frac{h_{\Gamma}^{m+1 / 2}}{h_{\Omega}^{k-1}} \leqslant \frac{h_{\Gamma}^{p}}{\left(C_{s}\right)^{k-1}}
$$

where $p=m-k+3 / 2-s(k-1)>0$. To estimate $|\lambda|_{m} /\|\varphi\|_{k}$, recall that $\varphi$ is an eigenvector associated with the eigenvalue $\xi$ of the operator $S$ introduced in Lemma 4. The operator $S$ is a bounded map from $H^{k}(\Omega)$ into $H^{k+2}(\Omega) \cap H_{0}^{1}(\Omega)$, thus $\xi\|\varphi\|_{k+2}=\|S \varphi\|_{k+2} \leqslant C_{k}\|\varphi\|_{k}$. Since $\lambda=\partial \varphi / \partial n$, and $k+1 / 2 \geqslant m$,

$$
|\lambda|_{m}=\left|\frac{\partial \varphi}{\partial n}\right|_{m} \leqslant\left|\frac{\partial \varphi}{\partial n}\right|_{k+1 / 2} \leqslant \hat{C}_{\tau}\|\varphi\|_{k+2} \leqslant \frac{1}{\xi} \hat{C}_{\tau} C_{k}\|\varphi\|_{k},
$$

where $\hat{C}_{\tau}$ is the trace constant for the trace map $H^{k+2}(\Omega) \stackrel{\partial / \partial n}{\longrightarrow} H^{k+1 / 2}(\Gamma),[10]$. Therefore, $|\lambda|_{m} /\|\varphi\|_{k} \leqslant \omega C_{\tau} C_{k}$ and so

$$
w(h) \leqslant \frac{\omega C_{\Gamma} \hat{C}_{\tau} C_{k}}{\hat{C}_{\Omega}\left(C_{s}\right)^{k-1}} h_{\Gamma}^{p}
$$

It follows that $w(h) \rightarrow 0$ as $h \rightarrow 0$ and (2.8) holds, in fact

$$
\inf _{\eta \in S_{h}(\Gamma)}|\lambda-\eta|_{-1 / 2} \leqslant w(h) \inf _{\chi \in S_{h}(\Omega)}\|\varphi-\chi\|_{1} \leqslant w(h)\left\|\varphi-\varphi_{h}\right\|_{1} .
$$

The proof of (2.12) will follow from (2.9) and a suitable lower bound for $\left\|\varphi-\varphi_{h}\right\|_{1}^{2}$ in terms of $\epsilon_{h}^{2}$. By (2.10),

$$
\left\|\varphi-\varphi_{h}\right\|_{1}^{2} \geqslant \inf _{\chi \in S_{h}(\Omega)}\|\varphi-\chi\|_{1}^{2} \geqslant \hat{C}_{\Omega}^{2} h_{\Omega}^{2 k-2}\|\varphi\|_{k}^{2} .
$$

Recall that $\Phi=(\varphi, \lambda)$ is chosen so that $A(\Phi, \Phi)=a(\varphi, \varphi)=1$, thus $1=a(\varphi, \varphi) \leqslant$ $C_{0}\|\varphi\|_{1}^{2}$, where $C_{0}=\max \left(1, c_{0}\right)$. Consequently, $\|\varphi\|_{k}^{2} \geqslant\|\varphi\|_{1}^{2} \geqslant 1 / C_{0}$ and $\left\|\varphi-\varphi_{h}\right\|_{1}^{2} \geqslant \hat{C}_{\Omega}^{2} h_{\Omega}^{2 k-2} / C_{0}$. On the other hand, by (1.7) and (1.8),

$$
\epsilon_{h}^{2} \leqslant \sup _{\substack{\Phi \in R(E) \\\|\Phi\|=1}}\left\{C_{\Omega}^{2} h_{\Omega}^{2 k-2}\|\varphi\|_{k}^{2}+C_{\Gamma}^{2} h_{\Gamma}^{2 m+1}|\lambda|_{m}^{2}\right\} \leqslant C(\omega) h_{\Omega}^{2 k-2},
$$

where

$$
C(\omega)=\sup _{\substack{\Phi \in R(E) \\\|\Phi\|=1}}\left\{C_{\Omega}^{2}\|\varphi\|_{k}^{2}+C_{\Gamma}^{2} C_{s}^{2-2 k} h_{\Gamma}^{2 p}|\lambda|_{m}^{2}\right\}
$$


It follows that $\left\|\varphi-\varphi_{h}\right\|_{1}^{2} \geqslant \hat{C}_{\Omega}^{2} \epsilon_{h}^{2} / C_{0} C(\omega)$.

Finally, by (2.9)

$$
\xi-\xi_{i}(h) \geqslant \xi\left\{\beta_{0}(h)\left\|\varphi-\varphi_{h}\right\|_{1}^{2}-\beta_{1}(h) \epsilon_{h}^{2}\right\} \geqslant \xi\left\{\beta_{0}(h) \frac{\hat{C}_{\Omega}^{2}}{C_{0} C(\omega)}-\beta_{1}(h)\right\} \epsilon_{h}^{2} .
$$

Since $\beta_{0}(h) \rightarrow \beta_{0}$ and $\beta_{1}(h) \rightarrow 0$, there is, for $h$ sufficiently small, a positive constant $\beta_{3}$ such that $\xi-\xi_{i}(h) \geqslant \xi \beta_{3} \epsilon_{h}^{2}$.

Corollary 1. Let $\omega$ be an eigenvalue of (1.1), (1.2) of multiplicity $m$, and let $\omega_{i}(h)$ be any one of the $m$ eigenvalues (counted according to multiplicity) of (1.5) (or (1.6)) converging to $\omega$. Then for $h$ sufficiently small, $\omega_{i}(h) \geqslant \omega$ and the estimate

$$
\omega_{i}(h)-\omega \geqslant \omega_{i}(h) \beta_{3} \epsilon_{h}^{2} \geqslant \omega \beta_{3} \epsilon_{h}^{2}
$$

holds.

Remark 1 . The restriction $k+1 / 2 \geqslant m$ in Theorem 3 is not really necessary. It just provides a convenient way to estimate $|\lambda|_{m} /\|\varphi\|_{k}$. If $k+2 j+1 / 2 \geqslant m$, for some nonnegative integer $j$, then $|\lambda|_{m} /\|\varphi\|_{k} \leqslant \widetilde{C}_{\tau} \cdot C(j) \omega^{j+1}$, where the constant $C(j)$ on the bounds for $S$ as a map from $H_{k+2 j}(\Omega)$ into $H_{k+2(j+1)}(\Omega) \cap H_{0}^{1}(\Omega)$.

Remark 2. The constant $C(\omega)$ in the proof of Theorem 3 decreases to $\sup _{\Phi \in R(E) ;\|\Phi\|=1} C_{\Omega}^{2}\|\varphi\|_{k}^{2}$ as $h \rightarrow 0$. This latter term can be estimated above by $C_{\Omega}^{2} \widetilde{C}^{2}(r) \omega^{2 r}$, where $r=\min \{l: 2 l+1 \geqslant k\}$ and $\widetilde{C}(r)$ is the product of the bounds for $S$ as a map from $H^{2 k-1}(\Omega)$ into $H^{2 l+1}(\Omega) \cap H_{0}^{1}(\Omega)$ for $l=1, \ldots, r$.

3. The Finite Dimensional Problem. In this section the matrix eigenvalue problem (1.6) is analyzed. It is shown that, despite the fact that the matrices in (1.6) are $(n+m) \times(n+m)$, the eigenvalues can be computed using an $n \times n$ nonnegative definite eigenvalue problem $u=\omega F_{1} u$. This problem yields $n-m$ positive eigenvalues $\omega$ and $m$ infinite eigenvalues corresponding to the null space of the matrix $F_{1}$. A scheme is presented for constructing the $n \times n$ matrix $F_{1}$ from the given matrices $K$, $M$, and $B$.

Consider then the eigenvalue problem

$$
\left[\begin{array}{ll}
K & M \\
M^{H} & 0
\end{array}\right]\left[\begin{array}{l}
u \\
\lambda
\end{array}\right]=\omega\left[\begin{array}{ll}
B & 0 \\
0 & 0
\end{array}\right]\left[\begin{array}{l}
u \\
\lambda
\end{array}\right],
$$

where $K$ and $B$ are positive definite Hermitian matrices of order $n \times n$, and $M$ is an $n \times m$ matrix. Thus, (3.1) is of order $(n+m) \times(n+m)$. Since $B$ is positive definite and Hermitian, it can be factored in the form $L L^{H}$, where $L$ is lower triangular and has positive elements on its diagonal. Thus, if (3.1) is multiplied on the left by

$$
\left[\begin{array}{cc}
L^{-1} & 0 \\
0 & I
\end{array}\right]
$$

and on the right by

$$
\left[\begin{array}{cc}
L^{-H} & 0 \\
0 & I
\end{array}\right]
$$


the equivalent eigenvalue problem

$$
\left[\begin{array}{cc}
G & J \\
J^{H} & 0
\end{array}\right]\left[\begin{array}{l}
v \\
\mu
\end{array}\right]=\omega\left[\begin{array}{ll}
I & 0 \\
0 & 0
\end{array}\right]\left[\begin{array}{l}
v \\
\mu
\end{array}\right]
$$

results, where $G=L^{-1} K L^{-H}, J=L^{-1} M$, and

$$
\left[\begin{array}{cc}
L^{-H} & 0 \\
0 & I
\end{array}\right]\left[\begin{array}{l}
v \\
\mu
\end{array}\right]=\left[\begin{array}{l}
u \\
\lambda
\end{array}\right] .
$$

It follows from Lemma $1,(1.10)$, that the matrix

$$
\left[\begin{array}{ll}
K & M \\
M^{H} & 0
\end{array}\right]
$$

is nonsingular and so

$$
\left[\begin{array}{ll}
G & J \\
J^{H} & 0
\end{array}\right]
$$

is also nonsingular. Let

$$
\left[\begin{array}{ll}
F_{1} & F_{2} \\
F_{2}^{H} & F_{3}
\end{array}\right]
$$

denote the inverse of the latter matrix, where $F_{1}$ is a Hermitian $n \times n$ matrix, $F_{2}$ is an $n \times m$ matrix, and $F_{3}$ is a Hermitian $m \times m$ matrix. Multiplying (3.2) through by this inverse yields the equivalent eigenvalue problem

$$
\left[\begin{array}{ll}
I & 0 \\
0 & I
\end{array}\right]\left[\begin{array}{l}
v \\
\mu
\end{array}\right]=\omega\left[\begin{array}{ll}
F_{1} & 0 \\
F_{2}^{H} & 0
\end{array}\right]\left[\begin{array}{l}
v \\
\mu
\end{array}\right] .
$$

In component form (3.3) becomes $v=\omega F_{1} v$ and $\mu=\omega F_{2}^{H} v$. Thus, $\omega<\infty$ is an eigenvalue of (3.3) with eigenvector $[v, \mu]^{T}$ if and only if $\omega$ is an eigenvalue of

$$
v=\omega F_{1} v
$$

with eigenvector $v$, where $\mu=\omega F_{2}^{H} v$.

There is also an "infinite" eigenvalue to keep track of in (3.3) and (3.4). Set $\omega=1 / \xi$ in (3.3), multiply by $\xi$, and consider the resulting eigenvalue problems in $\xi$. It is easily seen that 0 is an eigenvalue of this problem with multiplicity $2 m$. In fact, the $m$-dimensional subspace $\left\{[0, \lambda]^{T}, \lambda\right.$ arbitrary $\}$ forms the space of eigenvectors corresponding to 0 ; and the $m$-dimensional subspace $\left\{[u, 0]^{T}, u \in N\left(F_{1}\right)\right\}$ forms the space of generalized eigenvectors of order 2 , where $N\left(F_{1}\right)$ is the null space of $F_{1}$ and has dimension $m$ (Lemma 9). Note that the eigenvalues and eigenvectors of (3.3) transformed in this way are the eigenvalues and eigenvectors of the operator $T_{h}$. If (3.4) is transformed in a similar way, 0 is an eigenvalue of multiplicity $m$ corresponding to the null space of $F_{1}$. 
Since the characteristic polynomial corresponding to (3.3) has order $n+m$ and that corresponding to (3.4) has order $n$, it follows that (3.3) and (3.4) have $n-m$ finite eigenvalues counted according to multiplicity. These $n-m$ eigenvalues as well as their multiplicities are the same for (3.3) and (3.4). Moreover, since $F_{1}$ is nonnegative definite (Lemma 8 ) these eigenvalues are positive. Finally, it follows that none of these $n-m$ eigenvalues can have ascent greater than one when considered as eigenvalues of (3.3), i.e., there are no generalized eigenvectors associated with them.

Despite the fact that the original problem (1.6) was of order $n+m$, it suffices to consider the order $n$ nonnegative definite problem (3.4). If the component $\mu$ of an eigenvector is wanted, all that is necessary is to set $\mu=\omega F_{2}^{H} v$, where $v$ is an eigenvector of (3.4) corresponding to $\omega$. The remainder of this section is devoted to investigating the properties of $F_{1}$ and $F_{2}^{H}$ and outlining a scheme for computing these matrices.

For any matrix $A, N(A)$ will denote the null space of $A$ and $R(A)$ the range or equivalently the column space of $A$. The symbol $\perp$ will denote orthogonal complementation in the standard inner product, $x^{H} y$, on $\mathbf{C}^{n}$. Finally, let $\left\{e_{i}\right\}$ be the standard basis for $\mathbf{C}^{n}, e_{i}=(0, \ldots, 0,1,0, \ldots, 0)^{T}$ with 1 in the $i$ th position.

By definition the matrices $F_{1}, F_{2}, F_{3}$ satisfy the relations
(a) $F_{1} G+F_{2} J^{H}=I$
(a') $G F_{1}+J F_{2}^{H}=I$;
(b) $F_{1} J=0$;
(b') $J^{H} F_{1}=0$;
(c) $F_{2}^{H} G+F_{3} J^{H}=0$;
(c') $G F_{2}+J F_{3}=0$;
(d) $F_{2}^{H} J=I$;
(d') $J^{H} F_{2}=I$.

For simplicity the order of the matrices is left implicit. For example in (d) $F_{2}^{H} J$ is $m \times m$.

Lemma 7. $P_{1} \equiv G F_{1}$ and $P_{2} \equiv J F_{2}^{H}$ are projections on $\mathbf{C}^{n} . P_{1}$ is a projection onto $R^{\perp}\left(G^{-1} J\right)$ along $R(J)$, and $P_{2}=I-P_{1}$.

Proof. Multiply (3.5a) on the right by $F_{1}$ and use (3.5b'). This gives $F_{1} G F_{1}=$ $F_{1}$ or $\left(G F_{1}\right)^{2}=G F_{1}$ and so $P_{1}=G F_{1}$ is a projection. Multiply (3.5d) on the left by $J$ and on the right by $F_{2}^{H}$. This gives $\left(J F_{2}^{H}\right)^{2}=J F_{2}^{H}$; and thus, $P_{2}=J F_{2}^{H}$ is a projection. Moreover, by (3.5a'), $P_{1}+P_{2}=I$.

By (3.5b) $P_{1} J=G F_{1} J=0$, and so $N\left(P_{1}\right) \supset R(J)$. On the other hand, suppose, $u \in R^{\perp}(J)=N\left(J^{H}\right)$. Then $P_{2}^{H} u=F_{2} J^{H} u=0$. Thus, $u \in N\left(P_{2}^{H}\right)=R\left(P_{1}^{H}\right)=N^{\perp}\left(P_{1}\right)$. It follows that $R^{\perp}(J) \subset N^{\perp}\left(P_{1}\right)$, and so $N\left(P_{1}\right)=R(J)$.

Since $P_{1}=G F_{1}$, and $G$ is nonsingular, it follows that $N\left(F_{1}\right)=N\left(P_{1}\right)=R(J)$, and $R\left(P_{1}\right)=N^{\perp}\left(P_{1}^{H}\right)=N^{\perp}\left(F_{1} G\right)=R^{\perp}\left(G^{-1} J\right)$.

LEMMA 8. The matrix $F_{1}$ is nonnegative definite.

Proof. $u^{H} F_{1} u=u^{H} G^{-1} P_{1} u=\left(P_{1} u\right)^{H} G^{-1} P_{1} u+\left(P_{2} u\right)^{H} G^{-1} P_{1} u$. But $\left(P_{2} u\right)^{H} G^{-1} P_{1} u=\left(G^{-1} P_{2} u\right)^{H} P_{1} u=\left(G^{-1} J F_{2}^{H} u\right)^{H} P_{1} u$, and by Lemma 7 this term is zero. Consequently, $u^{H} F_{1} u=\left(P_{1} u\right)^{H} G^{-1} P_{1} u>0$ unless $P_{1} u=0$ or equivalently $u \in$ $N\left(P_{1}\right)=N\left(F_{1}\right)$.

LEMma 9. $\operatorname{dim} N\left(F_{1}\right)=\operatorname{dim} R(J)=m$.

Proof. Suppose there is an $m \times 1$ vector $\mu$ such that $J u=0$. Since 


$$
\left[\begin{array}{ll}
G & J \\
J^{H} & 0
\end{array}\right]
$$

is nonsingular and

$$
\left[\begin{array}{ll}
G & J \\
J^{H} & 0
\end{array}\right]\left[\begin{array}{l}
0 \\
\mu
\end{array}\right]=\left[\begin{array}{l}
J \mu \\
0
\end{array}\right]=\left[\begin{array}{l}
0 \\
0
\end{array}\right],
$$

it follows that $\mu=0$. This implies that the $m$ columns of $J$ are linearly independent and so $\operatorname{dim} R(J)=m$, but from Lemma 7 we have $N\left(F_{1}\right)=R(J)$.

The matrix $F_{1}$ can be constructed from the matrices $G$ and $J$. From Lemma 7 $F_{1}=G^{-1} P_{1}$, so what is needed is a representation for the projection $P_{1}$ in terms of $G$ and $J$. As will be shown below, $P_{1}=I-J Y$, where $Y$ is an $m \times n$ matrix gotten by solving $n$ problems involving the $m \times m$ positive definite matrix $J^{H} G^{-1} J$. Thus, the major portion of the computation in constructing $F_{1}$ involves inverting $G$ and solving the $n$ problems to construct $Y$. It is possible that $F_{1}$ need not be fully constructed in order to compute its eigenvalues and eigenvectors.

Let $g_{i}=G^{-1} e_{i}$ denote the $i$ th column of $G^{-1}$. Suppose $y_{i}$ is the solution of the $m \times m$ problem: $J^{H} G^{-1} J y_{i}=J^{H} g_{i}$. Note that by Lemma $9, J^{H} G^{-1} J$ is positive definite. Finally, set $w_{i}=g_{i}-G^{-1} J y_{i}$. Equivalently, $G w_{i}=e_{i}-J y_{i}$. Let $W=\left[w_{1}\right.$, $\left.\ldots, w_{n}\right]$ and $Y=\left[y_{1}, \ldots, y_{n}\right]$. Then $G W=I-J Y$.

Lemma 10. $F_{1}=W, P_{1}=I-J Y, P_{2}=J Y$, and $F_{2}^{H}=Y$.

Proof. $G w_{i}=G g_{i}-J y_{i}=e_{i}-J y_{i}$. Thus, by (3.5b),

$$
F_{1} e_{i}=F_{1} G w_{i}+F_{1} J y_{i}=F_{1} G w_{i}=P_{1}^{H} w_{i} .
$$

But $w_{i}^{H} J=\left(g_{i}^{H}-y_{i}^{H} J^{H} G^{-1}\right) J=g_{i}^{H} J-y_{i}^{H} J^{H} G^{-1} J=0$. Since $P_{1}^{H}$ is a projection onto $R^{\perp}(J)$ by Lemma 7 , it follows that $F_{1} e_{i}=P_{1}^{H} w_{i}=w_{i}$ and so $F_{1}=W$. Consequently, $P_{1}=G F_{1}=G W=I-J Y$ and so $P_{2}=I-P_{1}=J Y$. Finally, $J Y=P_{2}=J F_{2}^{H}$; thus $J\left(Y-F_{2}^{H}\right)=0$ and so $Y=F_{2}^{H}$.

Remark 3. Although it is not needed for the eigenvalue problem, the matrix $F_{3}$ can also be computed using the approach given here. By $\left(3.5 \mathrm{c}^{\prime}\right), G F_{2}+J F_{3}=0$. Thus,

$$
J F_{3}=-G F_{2}=-G Y^{H}=-G\left(\left(J^{H} G^{-1} J\right)^{-1} J^{H} G^{-1}\right)^{H}=-J\left(J^{H} G^{-1} J\right)^{-1}
$$

and so $F_{3}=-\left(J^{H} G^{-1} J\right)^{-1}$. This approach also yields an alternate scheme for solving the source problem

$$
\left[\begin{array}{lr}
K & M \\
M^{H} & 0
\end{array}\right]\left[\begin{array}{l}
u \\
\lambda
\end{array}\right]=\left[\begin{array}{l}
f \\
g
\end{array}\right], \quad[9] .
$$

COROLlaRY 2. The eigenvalues and eigenvectors of (3.3) can be computed from the $n \times n$ nonnegative definite problem

$$
\begin{aligned}
& v=\omega G^{-1}(I-J Y) v, \\
& \mu=\omega Y v
\end{aligned}
$$


where $Y=\left(J^{H} G^{-1} J\right)^{-1} J^{H} G^{-1}$. This problem produces $n-m$ positive eigenvalues and $m$ infinite eigenvalues corresponding to $N\left(G^{-1}(I-J Y)\right)=R(J)$.

Department of Mathematics, Statistics, and Computer Science

The American University

Washington, D. C. 20016

1. I. BABUŠKA, “The finite element method with Lagrangian multipliers," Numer. Math., v. 20, 1973, pp. 179-192.

2. I. BABUŠKA \& A. K. AZIZ, "Survey lectures on the mathematical foundations of the finite element method," The Mathematical Foundations of the Finite Element Method with Ap. plications to Partial Differential Equations, Academic Press, New York, 1973, pp. 5-359.

3. J. H. BRAMBLE \& J. E. OSBORN, "Rate of convergence estimates for nonselfadjoint eigenvalue approximation," Math. Comp., v. 27, 1973, pp. 525-549.

4. J. H. BRAMBLE \& A. H. SCHATZ, "Rayleigh-Ritz-Galerkin methods for Dirichlet's problem using subspaces without boundary conditions," Comm. Pure Appl. Math., v. 23, 1970, pp. 653-675.

5. N. DUNFORD \& J. T. SCHWARTZ, Linear Operators. II, Interscience, New York, 1963.

6. G. M. FIX, "Eigenvalue approximation by the finite element method," Advances in Math., v. 10, 1973, pp. 300-316.

7. G. M. FIX, "Hybrid finite element methods," SIAM Rev., v. 18, 1976.

8. W. G. KOLATA, "Approximation in variationally posed eigenvalue problems," Numer. Math., v. 29, 1978, pp. 159-171.

9. W. G. KOLATA, "On solving a matrix problem arising in a hybrid finite element method." (Preprint.)

10. J. NEČAS, Les Méthodes Directes en Théorie de Equations Elliptiques, Masson, Paris, 1967.

11. J. NITSCHE, "A projection method for Dirichlet problems using subspaces with nearly zero boundary conditions." (Preprint.)

12. J. NITSCHE, "Über ein Variationsprincip zur Lösung von Dirichlet-Problemen bei Verwendung von Teilräumen, die keinen Randbedingungen unterworfen sind," Abh. Math. Sem. Univ. Hamburg, v. 37, $1970 / 71$.

13. J. E. OSBORN, "Spectral approximation for compact operators," Math. Comp., v. 29, 1975 , pp. $712-725$. 